\title{
Imaging of Light Atoms in the Presence of Heavy Atomic Columns
}

\author{
M.P. Oxley,*** H.J. Chang,* A.Y. Borisevich,* M. Varela,* and S.J. Pennycook* \\ * Materials Science and Technology Division, Oak Ridge National Laboratory, PO Box 2008, Oak \\ Ridge, TN 37831 \\ ** Department of Physics and Astronomy, Vanderbilt University, Nashville, TN 37240
}

\begin{abstract}
Aberration correction in the scanning transmission electron microscope (STEM) has led to increased sensitivity and the ability to detect light atoms using annular dark field (ADF) imaging [1]. However, in most cases this requires the light atomic columns to be separated from heavier columns by a significant distance. Here we use Bloch wave simulations [2] to examine the visibility of light atoms situated close to heavier atomic columns using both $\mathrm{ADF}$ and electron energy loss spectroscopy (EELS).
\end{abstract}

In Fig. 1(a) we show an ADF image of $\mathrm{BiFeO}_{3}$ oriented in the $[001]_{\mathrm{rh}}$ zone axis taken using ORNL's aberration corrected VG Microscopes HB603U at $300 \mathrm{keV}$ incident energy. Because ADF intensity varies approximately with the square of the atomic number $Z$, the brightest features are the $\mathrm{Bi}$ columns with the Fe columns between them not clearly resolved. The $\mathrm{O}$ columns are not visible. The obvious feature of the image is that the Bi columns are not round. This is not a scan distortion, as alternate columns are distorted in opposite directions. This distortion may be understood if we look at the projected structure seen in Fig. 1(b). Each Bi column has a "satellite" $\mathrm{O}$ atom in close proximity, showing the same alternating pattern. In order to understand the primary factors behind the formation of these lobes, we consider the basic cross section for inelastic scattering.

$$
I(\mathbf{R}) \propto \int_{\text {all space }}|\psi(\mathbf{r}, \mathbf{R})|^{2} V_{\text {abs }}(\mathbf{r}) d \mathbf{r}
$$

Here $\mathbf{R}$ is the probe position, $\psi(\mathbf{r}, \mathbf{R})$ the real space wave function of the elastically scattered electron (attenuated due to thermal diffuse scattering) and $V_{\text {abs }}(\mathbf{r})$ the inelastic scattering potential. In Fig. 1(c) we show an ADF simulation with the satellite $\mathrm{O}$ atoms removed. As expected the $\mathrm{Bi}$ columns are now round. In Fig. 1(d) we include the $\mathrm{O}$ atoms in the calculation of $\psi$ but not in $V_{\text {abs }}(\mathbf{r})$. The column is distorted, so in this case the channeling of the incident electron has a significant role to play. In order to investigate whether the additional ADF scattering due to the $\mathrm{O}$ atoms plays a role, we show an ADF simulation without the $\mathrm{O}$ atoms in the calculation of $\psi$ but included in $V_{\text {abs }}(\mathbf{r})$. No distortion of the column is evident, so clearly the lobes shown in the full calculation in Fig. 1(f) are due to channeling rather than additional scattering.

An alternative way of imaging light columns is atomic resolution STEM EELS which measures an unambiguous signal which may be directly related to light atomic columns. In Fig. 2(a) we show an integrated O K-shell EELS image taken using a Nion UltraSTEM operating at $60 \mathrm{kV}$ for $\mathrm{LaMnO}_{3}$ in the [010] zone axis orientation. The isolated $\mathrm{O}$ columns are clearly resolved, but the La/O columns are actually at the position of the minimum signal, despite them containing $50 \%$ of the $\mathrm{O}$ atoms. This is also evident in the simulation (with projected structure inset) shown in Fig. 2(b). In Figs. 2(c) and (d) we show the contributions from the $\mathrm{O}$ and $\mathrm{La} / \mathrm{O}$ columns respectively. The La/O column provides at best $25 \%$ of the $\mathrm{O}$ column intensity which occurs between the $\mathrm{La} / \mathrm{O}$ columns. On the La/O columns the contribution is closer to $10 \%$. Clearly the heavier La atoms are leading to 
large angle scattering beyond the EELS detector and reduced intensity on the La/O columns. Atomic occupancy estimates, based on atomic resolution EELS, hence require a cautious approach.

\section{References}

[1] O.L. Krivanek et al., paper accepted by Nature, 2010.

[2] L.J Allen et al., Ultramicroscopy 96 (2003) 47.

[3] The research at ORNL was supported by the DOE Office of Basic Sciences, Division of Materials Sciences and Engineering and by appointment (HJC) to the ORNL Postdoctoral Research Program administered jointly by ORNL and ORISE.
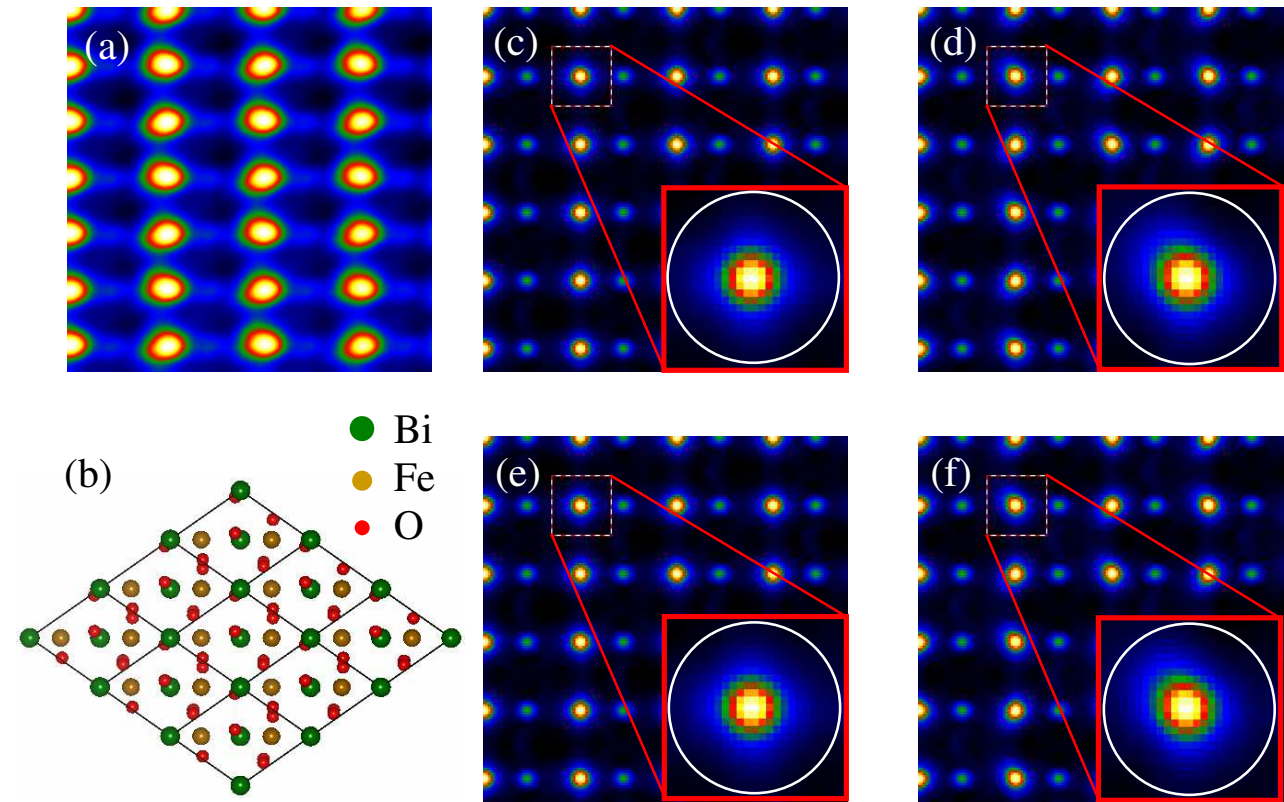

FIG. 1. ADF image of $\mathrm{BiFeO}_{3}$ in the $[001]_{\mathrm{rh}}$ zone axis orientation. (a) Experimental image acquired on the VG Microscopes HB603U fitted with a Nion aberration corrector. Image was corrected for nonorthogonal scan distortion and Fourier filtered. (b) Projected structure. (c) Simulated image with the $\mathrm{O}$ atoms next to the Bi columns removed. (d) Same as (c) but with the $\mathrm{O}$ atoms included in the calculation of the elastic wave function. (e) Same as (c) but with the ADF scattering potential of the $\mathrm{O}$ atoms included. (f) Full calculation.

FIG. 2. Integrated oxygen K-shell EELS signal from $\mathrm{LaMnO}_{3}$ in the [010] zone axis orientation. (a)
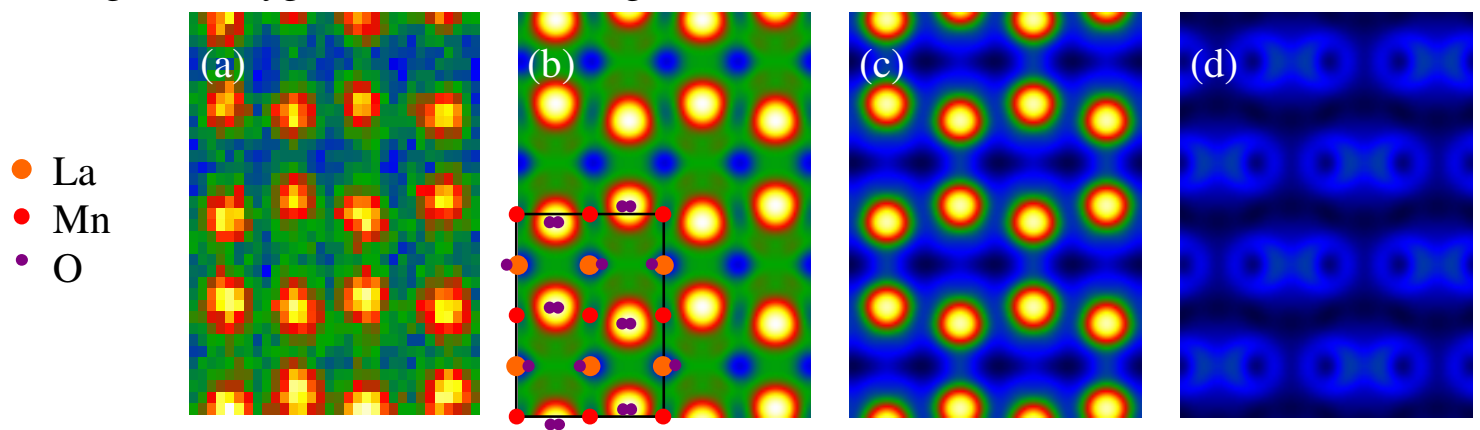

Experimental image acquired on the Nion UltraSTEM operating at $60 \mathrm{kV}$. Image was corrected for scan distortions. (b) Simulated image with projected structure inset. (c) Contribution to the total image from the isolated $\mathrm{O}$ columns. (d) Contribution to the total image from the $\mathrm{O}$ atoms on the $\mathrm{La} / \mathrm{O}$ columns. 